\title{
Antipsychotics: is it time to introduce patient choice?
}

\author{
Anthony P. Morrison, Paul Hutton, David Shiers and Douglas Turkington
}

\section{Summary}

Evidence regarding overestimation of the efficacy of antipsychotics and underestimation of their toxicity, as well as emerging data regarding alternative treatment options, suggests it may be time to introduce patient choice and reconsider whether everyone who meets the criteria for a schizophrenia spectrum diagnosis requires antipsychotics in order to recover.

\section{Declaration of interest}

A.P.M. and D.S. are both members of two National Institute for Health and Clinical Excellence guideline development groups: Psychosis and Schizophrenia in Children and Young People, and Psychosis and Schizophrenia in Adults (partial update).
Anthony Morrison (pictured) is Professor of Clinical Psychology at the University of Manchester and Associate Director of Clinical Research at Greater Manchester West Mental Health NHS Foundation Trust. He is involved in the development, evaluation and implementation of psychological approaches to the understanding and treatment of psychosis. Paul Hutton is a clinical psychologist based in the Psychosis Research Unit of Greater Manchester West Mental Health NHS Foundation Trust, where he is involved in developing psychological treatments for people with psychosis who are not taking antipsychotics, or who have continued difficulties despite taking them. David Shiers is Chair of the National Early Intervention Leads Network, Initiative to Reduce the Impact of Schizophrenia (IRIS), and is a Trustee of Rethink Mental Illness. His current research interests focus on the physical well-being of people with psychosis. Douglas Turkington is Professor of Psychosocial Psychiatry at Newcastle University. He prescribes antipsychotic medication and uses cognitive-behavioural therapy and other psychosocial interventions to work with service users with schizophrenia spectrum disorders.

It is evident that in mental health services worldwide there is an overreliance on antipsychotic medication in the treatment of schizophrenia and related disorders, which often leads to polypharmacy with incremental side-effect burden despite little evidence of improved efficacy. In the UK there has typically been little or no choice offered to service users who meet criteria for such diagnoses, with extensive use of coercion in decisions about medication. This is despite National Health Service (NHS) policy that actively promotes patient-led care, collaborative decisionmaking and provision of choice. In the context of emerging evidence regarding the overestimation of the effectiveness of antipsychotics and the underestimation of their toxicity, as well as emerging data regarding the possibility of alternative treatments, it may be time to reconsider the prevailing opinion that all service users with psychosis require antipsychotic medication in order to recover.

\section{Effectiveness of antipsychotics}

Recent evidence from systematic reviews and meta-analyses suggests that the efficacy and effectiveness of antipsychotics to produce clinically meaningful benefits for people with psychotic disorders have been overestimated. A meta-analysis showed that although there may be demonstrable effects of antipsychotics in comparison with placebo, the improvements over placebo are not as great as previously thought: ${ }^{1}$ the average change in symptoms rated with the Positive and Negative Syndrome Scale (PANSS) attributable to antipsychotics did not meet an empirically derived threshold for minimal clinical improvement, ${ }^{2}$ and only $17-22 \%$ experienced an important benefit (significant improvement or prevention of relapse) which could be attributed to the drugs rather than to placebo effects or natural recovery. A subsequent systematic review concluded that the improvements claimed for antipsychotics, old and new, are of questionable clinical relevance, ${ }^{3}$ with most trials failing to demonstrate even minimal improvement measured using the PANSS. There is also growing recognition that there is no discernible difference in effectiveness between first- and second-generation antipsychotics, supported by evidence from a recent meta-analysis. ${ }^{4}$ It is also relevant that there is evidence from double-blind trials in healthy volunteers that antipsychotic medication can result in secondary negative symptoms. ${ }^{5}$

\section{Adverse effects of antipsychotics}

There is also evidence, again from systematic reviews and metaanalyses as well as from large controlled studies, to suggest that the adverse effects of antipsychotics have been underestimated. For example, a recent systematic review concluded that some of the structural abnormalities in brain volume previously attributed to the syndrome of schizophrenia may be the result of antipsychotic medication. ${ }^{6}$ There is also considerable evidence that antipsychotics are associated with an increased risk of sudden cardiac death, ${ }^{7}$ and that some of the increased mortality observed in people with a diagnosis of schizophrenia is attributable to antipsychotic medication; ${ }^{8}$ increased cardiovascular risk is even detectable after the first exposure to any antipsychotic medication. ${ }^{9}$ There is indisputable evidence regarding weight gain induced by antipsychotics, ${ }^{10}$ which is also likely to be relevant to cardiovascular risk and mortality.

\section{Risk-benefit ratios, informed choice and collaborative decisions}

Given that mental health services appear to have overestimated the strength of the evidence base for antipsychotic medication, while underestimating the seriousness of the adverse effects, it seems sensible to re-evaluate the risk-benefit ratio of such drugs. This risk-benefit profile may be a factor in the high rates of non-adherence and discontinuation of medication found in patients with psychosis; thus, some decisions to refuse or discontinue antipsychotic medication may represent a rational informed choice rather than an irrational decision due to lack of insight or symptoms such as suspiciousness. Given accurate and honest assessments of both risks and benefits, it should be possible to prescribe antipsychotics in a more thoughtful and collaborative way, and these considerations should involve explicit discussion of the possibility of not prescribing at all. Provision of such choices may help to engage people who might otherwise 
reject services; for example, patients with low levels of insight and/ or high levels of internalised stigma might resist medication but consider that talking to someone is acceptable.

To facilitate informed choice and decision-making, we require a much better evidence base to help address questions such as how and when medication might be required, who is most likely to respond and what alternatives exist. There is some evidence for different trajectories of response, with a small proportion of patients demonstrating a rapid and dramatic favourable response to certain antipsychotics, ${ }^{11}$ but more research is clearly required to inform our ability to predict those most (and least) likely to respond to antipsychotics. Shorter duration of untreated psychosis has been shown to be a predictor of response to antipsychotics, ${ }^{12}$ which could be employed as an argument against offering no medication as a choice. However, any additional benefits of early treatment would still need to be evaluated against the long-term risks, and the traditional assumption that 'untreated psychosis' can only be treated by prescribing antipsychotics (and therefore not by psychosocial therapies) has yet to be comprehensively tested. It is relevant to this assumption that 20 -year outcome data from the Chicago Follow-Up Study suggest that service users who decide not to take antipsychotics (often against medical advice) do relatively well, if not better, in comparison with service users who take such medication continuously. ${ }^{13}$

In addition to research regarding predictors of response to antipsychotics, research is also required to inform evidence-based alternatives to antipsychotic medication, since the most likely candidates (such as psychosocial treatments including cognitive therapy and family interventions) have almost exclusively been evaluated as an adjunct to medication. There are a few exceptions, such as a recent trial of cognitive therapy for people who chose not to take antipsychotics; ${ }^{14}$ however, more clinical trials with greater methodological rigour are clearly needed.

It may be time to reappraise the assumption that antipsychotics must always be the first line of treatment for people with psychosis; rather, this should be a collaborative decision that is balanced with provision of informed choices and the offer of evidence-based alternatives. These decisions should be negotiated with service users on the basis of the likely positive and negative consequences and the prioritisation of their goals and values; such a collaborative approach might also result in better response for those who choose to take antipsychotics, since the quality of relationship with the prescribing clinician is associated with attitudes and adherence to medication. ${ }^{15}$

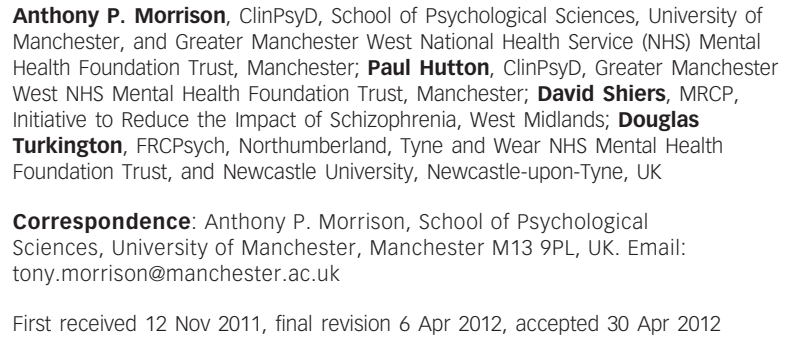

\section{References}

1 Leucht S, Arbter D, Engel RR, Kissling W, Davis JM. How effective are secondgeneration antipsychotic drugs? A meta-analysis of placebo-controlled trials. Mol Psychiatry 2009; 14: 429-47.

2 Leucht S, Kane JM, Etschel E, Kissling W, Hamann J, Engel RR. Linking the PANSS, BPRS, and CGI: clinical implications. Neuropsychopharmacology 2006; 31: 2318-25.

3 Lepping P, Sambhi RS, Whittington R, Lane S, Poole R. Clinical relevance of findings in trials of antipsychotics: systematic review. Br J Psychiatry 2011; 198: $341-5$.

4 Leucht S, Corves C, Arbter D, Engel RR, Li C, Davis JM. Second-generation versus first-generation antipsychotic drugs for schizophrenia: a metaanalysis. Lancet 2009; 373: 31-41.

5 Artaloytia JF, Arango C, Lahti A, Sanz J, Pascual A, Cubero P, et al. Negative signs and symptoms secondary to antipsychotics: a double-blind, randomized trial of a single dose of placebo, haloperidol and risperidone in healthy volunteers. Am J Psychiatry 2006; 163: 488-93.

6 Moncrieff J, Leo J. A systematic review of the effects of antipsychotic drugs on brain volume. Psychol Med 2010; 40: 1409-22.

7 Ray WA, Chung CP, Murray KT, Hall K, Stein CM. Atypical antipsychotic drugs and the risk of sudden cardiac death. N Engl J Med 2009; 360: $225-35$.

8 Weinmann S, Read J, Aderhold V. Influence of antipsychotics on mortality in schizophrenia: systematic review. Schizophr Res 2009; 113: 1-11.

9 Foley DL, Morley KI. Systematic review of early cardiometabolic outcomes of the first treated episode of psychosis. Arch Gen Psychiatry 2011; 68: 609-16.

10 Álvarez-Jiménez M, Hetrick SE, González-Blanch C, Gleeson JF, McGorry PD. Non-pharmacological management of antipsychotic-induced weight gain: systematic review and meta-analysis of randomised controlled trials. $\mathrm{Br} J$ Psychiatry 2008; 193: 101-7.

11 Marques TR, Arenovich T, Agid O, Sajeev G, Muthen B, Chen L, et al. The different trajectories of antipsychotic response: antipsychotics versus placebo. Psychol Med 2011; 41: 1481-8.

12. Perkins $D$, Lieberman J, Gu H, Tohen $M$, McEvoy J, Green A, et al. Predictors of antipsychotic treatment response in patients with first-episode schizophrenia, schizoaffective and schizophreniform disorders. $\mathrm{Br} \mathrm{J}$ Psychiatry 2004; 185: 18-24.

13 Harrow M, Jobe TH, Faull RN. Do all schizophrenia patients need antipsychotic treatment continuously throughout their lifetime? A 20-year longitudinal study. Psychol Med 2012; Feb 17 (Epub ahead of print).

14 Morrison AP, Hutton $\mathrm{P}$, Wardle $\mathrm{M}$, Spencer $\mathrm{H}$, Barratt $\mathrm{S}$, Brabban $\mathrm{A}$, et al. Cognitive therapy for people with a schizophrenia spectrum diagnosis not taking antipsychotic medication: an exploratory trial. Psychol Med 2012; 42: 1049-56.

15 Day JC, Bentall RP, Roberts C, Randall F, Rogers A, Cattell D, et al. Attitudes toward antipsychotic medication: the impact of clinical variables and relationships with health professionals. Arch Gen Psychiatry 2005; 62 717-24. 